\title{
Properties and processes that influence CME geo-effectiveness
}

\author{
Benoit Lavraud ${ }^{1,2}$ and Alexis Rouillard ${ }^{1,2}$ \\ ${ }^{1}$ Institut de Recherche en Astrophysique et Planétologie, Université de Toulouse (UPS), \\ Toulouse, France \\ ${ }^{2}$ UMR 5277, Centre National de la Recherche Scientifique, Toulouse, France \\ email: benoit.lavraud@irap.omp.eu
}

\begin{abstract}
The geo-effectiveness of coronal mass ejections (CME) is determined by a complex chain of processes. This paper highlights this fact by first discussing the importance of CMEs intrinsic properties set at the Sun (e.g., trajectory, eruption process, orientation, etc.). We then review other key processes that may occur during propagation (e.g., shocks, compressions, magnetic flux erosion) and in the specific interaction with Earth's magnetosphere (e.g., magnetic properties, preconditioning mechanisms). These processes sequentially have a significant influence on the final geo-effectiveness of CMEs. Their relative importance is discussed. While the CME's trajectory, magnetic field orientation, velocity and their duration as set at the Sun certainly are key ingredients to geo-effectiveness, other processes and properties, that at first appear secondary, often may be as important.
\end{abstract}

Keywords. Plasmas - Sun: coronal mass ejections (CMEs) - Sun: solar-terrestrial relations Earth

\section{Introduction}

The impacts of solar phenomena, and in particular that of coronal mass ejections, in the heliosphere has been the focus of much attention in recent years. This theme is known under the general terminology of "Space Weather". It has been driven by the accumulation of evidences for significant societal and economical implications such as radiation hazard to astronauts, partial to full spacecraft power failures, GPS signal alterations, or ground power grid and transformer failures. It has led to numerous dedicated developments, including databases, analysis tools, virtual observatories, instruments and mission concepts. The purpose of the present paper is not to focus on these impacts, but rather to review the complex chain of physical processes that come into play for determining whether and how much a CME eruption may affect Earth environment.

Of particular importance are the solar wind velocity and the direction and strength of the interplanetary magnetic field, which combine into the Dawn-Dusk component of the solar wind electric field $\mathbf{E}=-\mathbf{V} \times \mathbf{B}$ (e.g., Gonzales et al. 1974, Siscoe \& Crooker 1974, Burton et al. 1975, Perreault et al. 1978). This is because the solar wind electric field relates to the global dayside magnetic reconnection rate. The role of these parameters was already made evident in early works that attempted to derive driving function of the magnetospheric response to solar wind conditions. In his review, Akasofu (1981) already noted 13 such coupling functions. Many more complex coupling functions have been devised since then. Recent ones can be found in Newell et al. (2007), Borovsky (2008), and Tenfjord \& Østgaard (2013). In addition to the electric field driver (often referred to as the $\mathrm{VBz}$ parameter in coupling functions), the duration of the driver is of course of paramount importance for cumulative energy input reasons. This cumulative 
effect needs to be accounted for when one implements coupling functions, e.g., such as the semi-empirical model of the ring current Dst index by O'Brien \& McPherron (2000) (based on Burton et al. 1975).

The central role of Coronal Mass Ejections (CMEs) in driving geomagnetic activity was early recognized, and well put into context in the work by Gosling (1993). Magnetic Clouds (MCs) are a subset of CMEs. These are defined by a flux rope structure with enhanced magnetic field and a clear and smooth rotation of its components, and a lowerthan-usual proton temperature (Burlaga et al. 1982). The fact that not all CMEs are observed as clear flux ropes (i.e., MCs) is generally attributed to the sampling geometry through the magnetic structure (e.g., Jian et al. 2006). For more details on the basic CME and MC properties, the reader is referred to the review by Démoulin (this issue; and references therein).

\section{Properties set at the Sun}

\subsection{Eruption location, trajectory and deflections}

The first key ingredient to assess the potential impact of a CME at Earth is of course its eruption location on the Sun and ensuing trajectory. The huge 23rd July 2012 CME recorded at STEREO-A is a good example with, despite the potential for a record geomagnetic storm, a trajectory at $\sim 120^{\circ}$ from the Sun-Earth line (Russell et al. 2013).

On the other hand, processes in the corona during and after ejection may alter the trajectory. First, significant latitudinal trajectory deflection may occur through interaction of the CME with the strong magnetic fields from the adjacent polar coronal holes. This was recently highlighted through global magnetohydrodynamic (MHD) modeling by Zuccarello et al. (2012), as is illustrated in Figure 1. An important implication of this process is that even CMEs which originate at high latitudes will tend to be channeled towards the ecliptic plane (along the helmet streamers and heliospheric current sheet; HCS). This phenomenon will thus statistically tend to focus CMEs toward the Earth's latitude. It should be noted, however, that this effect plays a role mainly during nonmaximum solar cycle periods when CMEs are generally isolated and the dipolar structure of the solar magnetic field is well in place. During solar maximum period, the much more complex structure of the streamers and active regions will tend to homogenize this effect in latitude.

In addition to latitudinal deflections, longitudinal deflections also occur for similar reasons; i.e., through interaction with low-latitude coronal holes in the presence of a warped
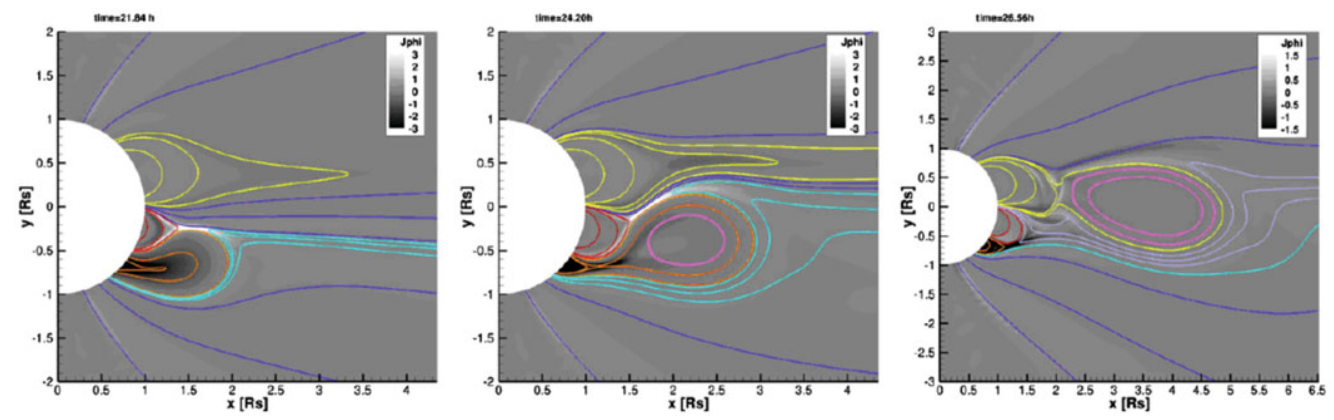

Figure 1. Three temporal snapshots of the evolution of a CME (orange and pink lines) erupting at low southern latitudes, from global MHD simulations. The CME is channeled towards lower latitudes and eventually propagates in the ecliptic plane. Adapted from Zuccarello et al. (2012). 
helmet streamer/HCS. As a result CMEs tend to be channeled along the Parker spiral direction during their ejection and further propagation. This is the explanation typically put forth to explain the trend for the origin of Earth-impacting CMEs to be statistically skewed towards the West side of the solar disk (cf. Zhang et al. 2003; but see also Cane et al. 2000 for earlier, contradicting results).

\subsection{Ejection processes and models: a spectrum of CME strengths and sizes}

The geo-effectiveness of CMEs directed towards Earth of course largely relies on the energetics of the eruptive phenomenon at the Sun. In other words, the potential geoeffectiveness of a CME directly depends upon three key parameters: the size of the $\mathrm{CME}$, its initial magnetic field strength and its bulk speed. The two latter set the VBz parameter (cf. introduction), while the former controls its duration. These directly impact the ability of the CME to drive strong coupling at Earth's magnetopause and in turn large-scale magnetospheric convection and a geomagnetic storm.

There exist various models of CME eruption, and inferred processes. The point worth conveying here is that there should exist a whole spectrum of processes involved, and thus also a whole spectrum of resulting CME strengths and sizes. We highlight this fact in Figure 2 by presenting what may be two extremes cases of this spectrum. Figures 2a and $2 \mathrm{~b}$ display solar disk images during a flare and CME event in November 1997, with Figure $2 \mathrm{~b}$ showing in addition reconstructed magnetic fields (using a coronal model). This work by Delannée \& Aulanier (1999) investigated an eruption model, called transequatorial filament eruption based on large-scale loops threading both the southern and northern hemispheres. This model, and inferred processes, thus involves very large-scale structures by definition. Resulting CMEs are expected large in size, and may possess strong internal energy (e.g., magnetic field and speed).

Figure $2 \mathrm{c}$ shows a schematic describing the release of plasmoid-type structures through reconnection at the tip of a helmet streamer (Wang et al. 2000). This type of CME release mechanism is unlikely to produce strong and fast CMEs. Studies have actually linked these plasmoids with the in situ observation of small-scale flux ropes with low magnetic field and speed entrained by the solar wind at 1 AU (e.g., Rouillard et al. 2009 and Rouillard et al. 2011). In conclusion, the transequatorial filament eruption model is more likely to produce geo-effective CMEs than the streamer blowout mechanism, and a whole range of models and associated processes exists in between.
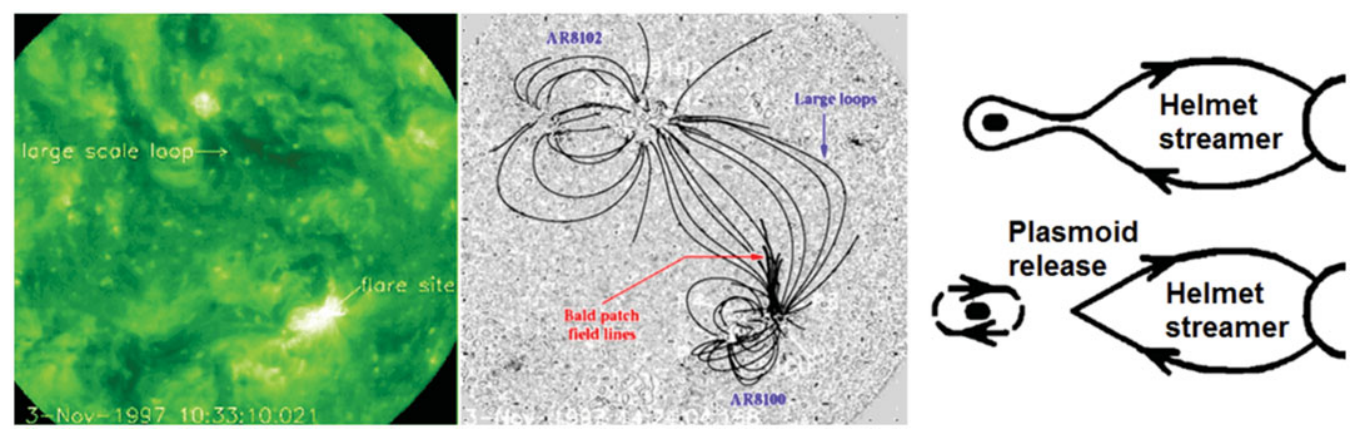

Figure 2. Illustrations of two drastically different CME eruption models: (a and b) the transequatorial filament eruption model (Figure courtesy of G. Aulanier; based on Delannée \& Aulanier (1999)) and c) the plasmoid release model (Figure adapted from Wang et al. (2000)). The former involves large regions of the Sun and large-scale processes, while the latter proposes the release of small-scale structures through reconnection at the tip of the helmet streamer. 


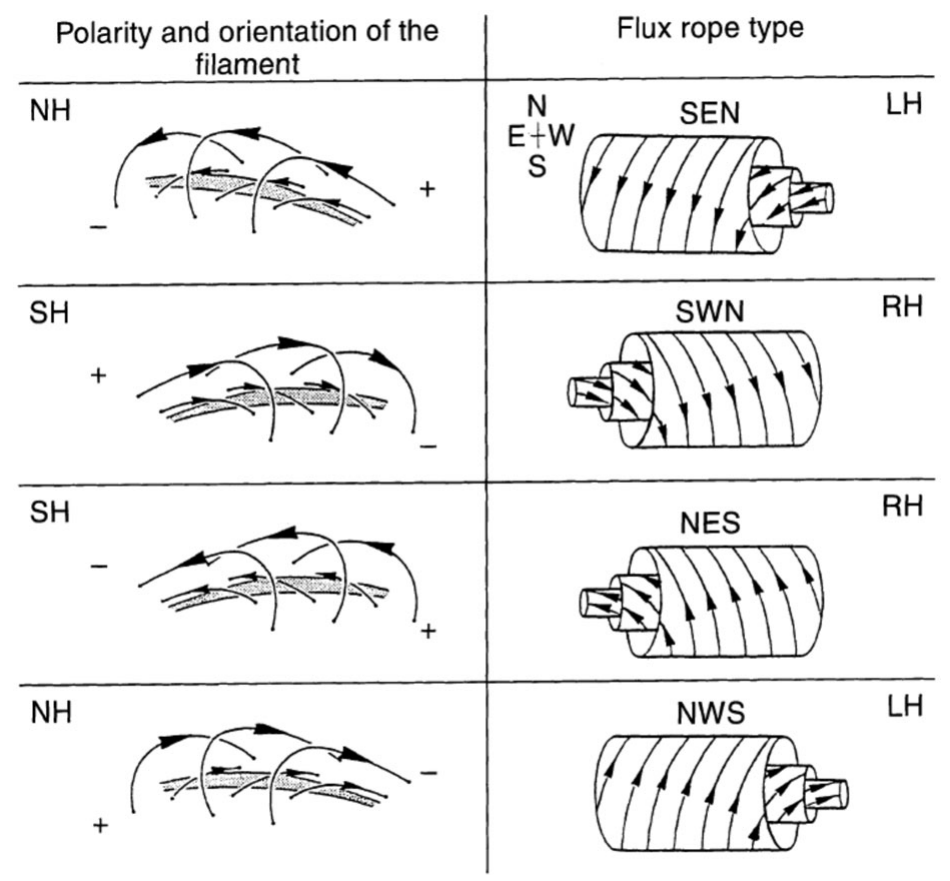

Figure 3. Relationship between the polarity and orientation of solar filaments and the associated CME flux rope structure, as deduced from a statistical study. From Bothmer \& Schwenn (1998).

\subsection{CME acceleration and deceleration in the corona}

It is known that CME speeds as observed near the Sun (e.g., from coronograph) hardly correspond with CME speeds measured in situ at 1 AU. This is particularly true for halo CMEs (directed towards Earth), where projection effects further render the CME speed hard to determine. This has direct implications for our ability to estimate CME transit times between the Sun and Earth (e.g., Gopalswamy et al. 2001 and references therein). Studies of CME acceleration using coronograph images alone show that strong CME acceleration or deceleration occur in the corona, with initially slow (fast) CMEs accelerating (decelerating) (e.g. Gopalswamy et al. 2000). CMEs interact with the ambient solar wind, so that the dichotomy between acceleration and deceleration is centered for CME speeds of order of that of the slow solar wind: $400 \mathrm{~km} / \mathrm{s}$ (Gopalswamy et al. 2000). This process is akin to general drag (e.g., Cargill et al. 1995). These models predict that most of the drag occurs close to the Sun, soon after eruption, and that CME speed is more constant past few tens of solar radii. Of course, these processes are very important since the final CME speed is a key parameter for geo-effectiveness.

\subsection{Magnetic flux rope orientation and polarity}

Because the sign and magnitude of the IMF Bz component is critical for the triggering of geomagnetic storms, as mediated by magnetic reconnection at the dayside magnetopause, the orientation of the CME (or MC) plays a key role. For CMEs that do not show a flux rope structure (cf. review by Démoulin (this issue)), or for sheaths ahead of CMEs, the magnetic field strength and direction is rather unordered and thus the geoeffectiveness hard to anticipate. However, in the case of well-formed flux rope structures such as in MCs, the orientation and magnetic polarity of active regions and filaments at the origin of CME eruption at the Sun directly impact the timing and intensity of the 
ensuing geomagnetic storm. This is because the polarity at the Sun generally determines (statistically) whether it is the leading or trailing portion of the CME that contains the southward-oriented magnetic field (e.g., Bothmer \& Schwenn 1998, cf. Figure 3). On the other hand, the tilt of the active region or filament, and other deflection/rotation processes in the corona, determine the MC main axis orientation in interplanetary space. The resulting latitudinal angle of the main flux rope axis orientation is particularly important because it can lead to a reduced southward Bz component at $1 \mathrm{AU}$. Often MCs at $1 \mathrm{AU}$ have such a large latitudinal angle that the $\mathrm{Bz}$ component is very weak and no geomagnetic storm is triggered despite the MC being intense and fast. Finally, it should be noted that whether MCs have a South-North or North-South magnetic polarity is solar cycle dependent (Mulligan et al. 1998). This may have an impact when propagation and compression effects come in, as discussed next in Section 3.

\section{Processes during propagation}

\subsection{CME expansion, drag, shocks and sheath}

Following eruption near Sun where most of the solar wind and CME acceleration occurs, numerous additional processes come into play for determining geo-effectiveness. The first to mention is the global expansion of the CME. While small-scale CMEs likely have insufficient internal magnetic fields for significant expansion, large-scale CMEs with intense magnetic fields are know to expand significantly in both the radial and longitudinal/latitudinal directions. The expansion has been shown to be often self-similar (Démoulin and Dasso 2009), and models that represent such global expansion have been devised (Owens 2006; Owens et al. 2006).

While CMEs that significantly expand in the interplanetary medium will see their internal magnetic field intensity decrease significantly, the decrease is less for CMEs that strongly interact with the ambient solar wind. CMEs that expand the most have lower fields but longer duration. In terms of geo-effectiveness, the two effects are competing but overall a large magnetic field remains the critical component to produce large storms. Statistics show that compressions and the formation of shocks, ahead of CMEs that strongly interact with the ambient solar wind (and thus expand less), are the source of major storms. This was already found in early studies (Gosling et al. 1990). While a separate review would be required to cover geo-effectiveness aspects related to energetic particles, it should be noted here that CME-driven shocks are a major source of such particles, in particular for the largest and fastest CMEs.

While the formation of shocks and sheaths produces enhanced magnetic fields, CMEs are subject to a drag force that slows them down as they expand into a slower solar wind. The drag is primarily dependent upon the speed difference between the CME and solar wind into which it expands. For fast CMEs modelling efforts typically show strong deceleration close to the Sun, then weaker drag and more constant speed further out in the heliosphere, although unusual CME speed, size or density can lead to unusual behaviours (e.g., Vrsnak et al. 2010; Subramanian et al. 2012). The impact of drag on CME speed at $1 \mathrm{AU}$ is very important, in particular in extreme cases, as speed strongly influences CME geo-effectiveness.

\subsection{HSS-CME and CME-CME interactions}

Interaction between CMEs and the ambient solar wind is not limited to the formation of a shock and sheath at its front. Interaction has often been reported at the rear of CMEs, for instance in the presence of a trailing high speed stream (HSS) emanating from a nearby coronal hole. The impact of such a configuration on geo-effectiveness was put forth in 

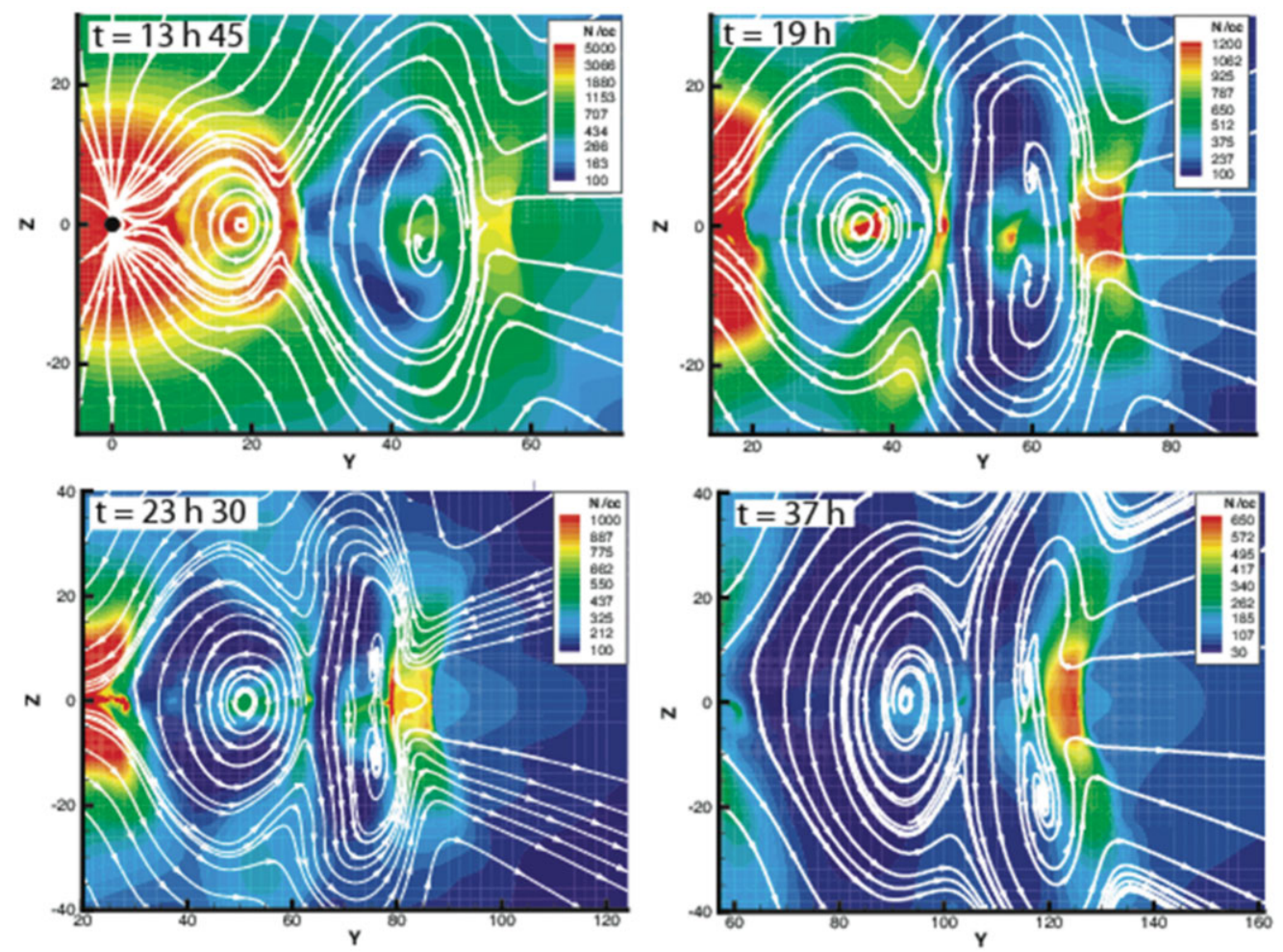

Figure 4. Snapshots of a 3D global MHD simulation for the case of CME-CME interaction at four times during the run by Lugaz et al. (2005). The faster CME (to the left) overcomes and compresses the first, slower CME. Magnetic reconnection and magnetic flux erosion are also likely to occur during such interaction.

the work by Fenrich \& Luhmann (1998), who showed that in the case of a North-South polarity MC (i.e., with a trailing southward Bz) the compression exerted by the HSS led to increased magnetic fields at the rear of the MC and enhanced geo-effectiveness.

Such rear compression may also occur in the context of direct CME-CME interaction, i.e., when a CME is overcome by a faster one launched only slightly later from the Sun. Such cases are not rare, in particular during solar cycle maximum. Such a complex interaction was studied by Lugaz et al. (2005), and is depicted in Figure 4. Strong compression regions are formed both in front of the first $\mathrm{CME}$ and at the interface between the two CMEs. The resulting compressed fields may enhance ensuing storm strength depending on the orientation and magnetic polarity of each MC (cf. Section 2.4). In addition to compression, CME-CME interaction may induce magnetic reconnection and magnetic flux erosion, as further discussed in Section 3.3, with implications for geo-effectiveness.

With the advent of the two STEREO spacecraft, orbiting the Sun ahead and behind the Earth on similar orbits, it has been possible to track CMEs all the way from the Sun to the Earth. This is thanks to observation locations away from the Sun-Earth line and the use of dedicated new instruments - the Heliospheric Imagers - which observe heliospheric electron density fluctuations from Thomson scattering in white light (these are essentially coronographs with wider fields-of-view). Because they capture density fluctuations, these instruments have been widely used to track the propagation of CMEs by observing the compression regions either in front or trailing CMEs (e.g., Rouillard et al. 2010). These novel capabilities have permitted to better constrain the origin of CMEs, 

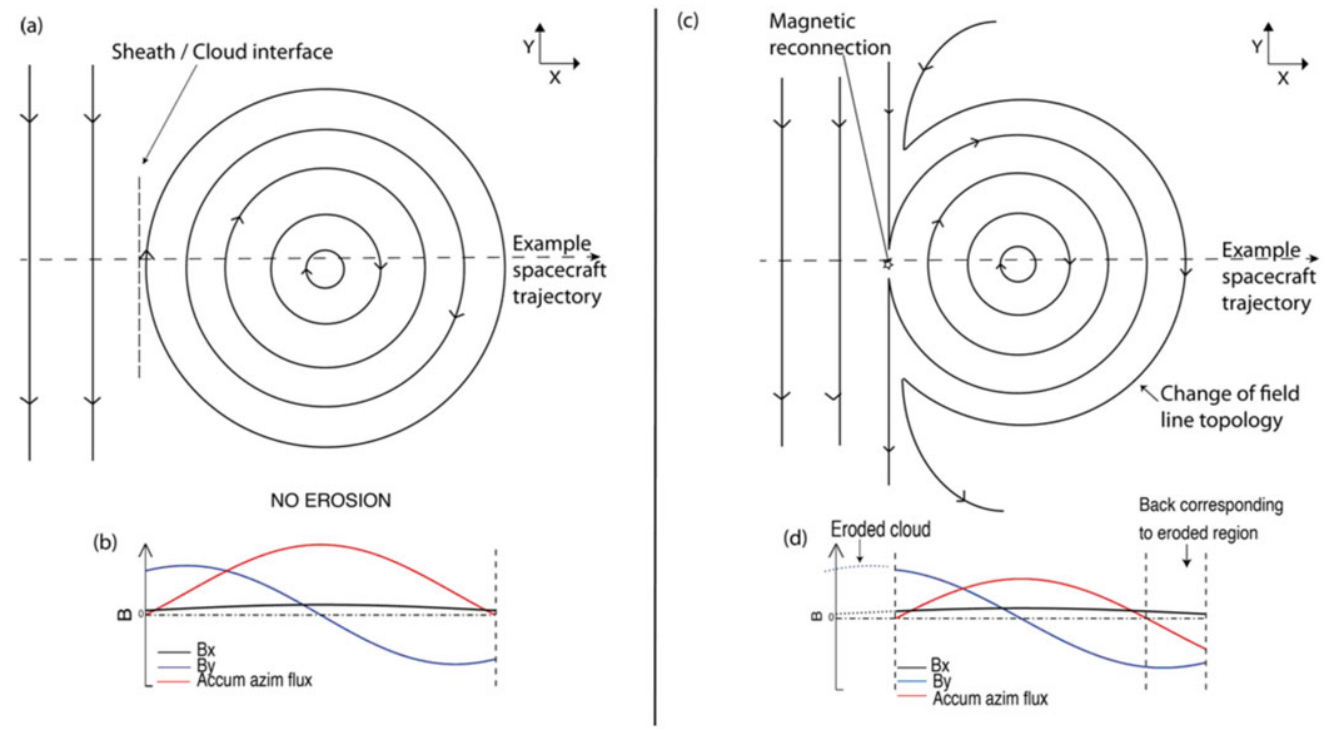

Figure 5. Schematic illustration of the impact of flux erosion by magnetic reconnection at the front of MCs. The magnetic structure and related magnetic field components in the MC frame for a non-eroded MC are shown in panels (a) and (b). Panels (c) and (d) show the structure that results from magnetic erosion at the front of a $\mathrm{MC}$, with an imbalance in azimuthal magnetic flux. From Ruffenach et al. (2012)

and to determine their trajectory and arrival time at Earth (or other locations) with unprecedented advance timing and reliability.

\subsection{Magnetic flux erosion by magnetic reconnection}

Despite a reduced speed owing to drag, we noted that compressions during CME propagation into the ambient solar wind typically induce enhanced fields and geo-effectiveness. In this Section we highlight an other mechanism that occurs during propagation, and which may diminish CME geo-effectiveness: magnetic flux erosion by magnetic reconnection.

The possibility of magnetic flux erosion at the front of CMEs dates back to McComas et al. (1988). It was further studied and quantified by Dasso et al. (2007) and Ruffenach et al. (2012), in particular. A basic implication of this mechanism is the bulk removal i.e., the erosion of the CME magnetic flux, at its front. This is observed as an imbalance in azimuthal magnetic flux when the magnetic field of the CME is analysed in the proper frame, relative to the flux rope orientation. This analysis may thus only be applied to CMEs that have a flux rope structure, i.e., MCs. This is schematically presented in Figure 5 , where the eroded MC on the right-hand side is characterized by an excess of magnetic flux at its rear. In addition to this signature in the magnetic field, Ruffenach et al. (2012) pointed out several other signatures of this process, including the observation of local magnetic reconnection at the MC front boundary and the observation of a distinct magnetic topology in the back region using suprathermal electrons.

Lavraud et al. (2013) recently used simple modelling to investigate the potential geoeffectiveness of this process. They suggested that the reconnection rates observed in the solar wind at $1 \mathrm{AU}$, despite appearing low at first glance, are overall consistent with the average reconnection rates required to produce the erosion calculated from the imbalance in azimuthal magnetic field (cf. also Ruffenach et al. 2012). For a MC with convenient 
South-North polarity and geometry, their analysis suggested an ensuing storm strength of order $30 \%$ lower in terms of the Dst index (using a simple semi-empirical model) as a consequence of magnetic flux erosion at its front.

\section{Specifics of the interaction with Earth's magnetosphere}

This is an extremely broad subject; we therefore focus only on few key aspects that highlight the importance of the basic characteristics of the object considered in determining the effectiveness of a CME to perturb its nearby environment. We mainly discuss the case of Earth, but comparisons with other bodies are given.

\subsection{Properties of the object impacted}

Firstly, the object size and the strength of its magnetic dipole are very important. This determines for instance whether the magnetopsheric dynamics is controlled by processes primarily internal (giant planets) or external (e.g., Mercury or mostly unmagnetized planets such as Mars) to the body. The case of Earth can be considered as driven both ways. A key parameter is then of course the magnetic dipole orientation. Because external driving depends upon the magnetic shear at the magnetopause, whether the dipole axis is directed northwards like at Earth or southwards like at Saturn plays a key role in assessing how a CME of given magnetic polarity (cf. Figure 3) will interact. The case of Uranus, with a dipole axis close to the ecliptic plane is in this context highly unusual.

The magnetic dipole strength, the presence of moons within the magnetosphere, the proximity to the Sun and the properties of the atmosphere-exosphere system are all very important in assessing the impact of internal process. This is true in particular with regards to ion escape/outflows (Mars, Earth, etc.) and the values of ionospheric conductivities (e.g., Earth versus Mercury). The latter for instance affects global magnetospheric convection, such as in the case of polar cap potential saturation at Earth (e.g., Shepherd et al. 2007).

\subsection{Basic paradigm for coupling at Earth}

The interaction with the interplanetary medium essentially occurs at the magnetopause for magnetized planets. Although other processes occur at this boundary, during strong coupling the key energy transfer mechanism is magnetic reconnection at the front (sunward-side) of the magnetosphere. At Earth the basic paradigm is that dayside reconnection leads to energy and momentum transport through the magnetopause, which in turn drives magnetospheric convection. This leads to energy loading in the magnetotail and ensuing release and transport towards the inner magnetosphere through yet other reconnection mechanisms (either distant or nearer to Earth). The magnetic flux is ultimately transported back towards the dayside into the reconnection region so as to close the magnetic cycle. This is known as the Dungey cycle (Dungey 1961).

Although complexities arise in the details of how the solar wind properties control the local and global rates of magnetic field reconnection at the dayside magnetosphere (e.g., Borovsky 2008), to first order the key parameters are the value of the magnetic field $\mathrm{Bz}$ component and the bulk speed (Gonzales et al. 1974; Siscoe \& Crooker 1974; Perreault et al. 1978; Newell et al. 2007). These combine into the Dawn-Dusk electric field, which is related to reconnection rates at the magnetopause through mapping into the dayside magnetic reconnection line. The reader is referred to Lavraud \& Borovsky (2008), Borovsky (2008) and Lopez et al. (2010) for further discussion on this topic.

As mentioned previously, the duration of the strong driver is a key parameter to assess global geo-effectiveness. Semi-empirical models are thus typically built to represent the 
Table 1. List of low Mach number solar wind effects at the Earth's magnetosphere and the current status of observational evidence and physical understanding for them. Table adapted from Lavraud \& Borovsky 2008.

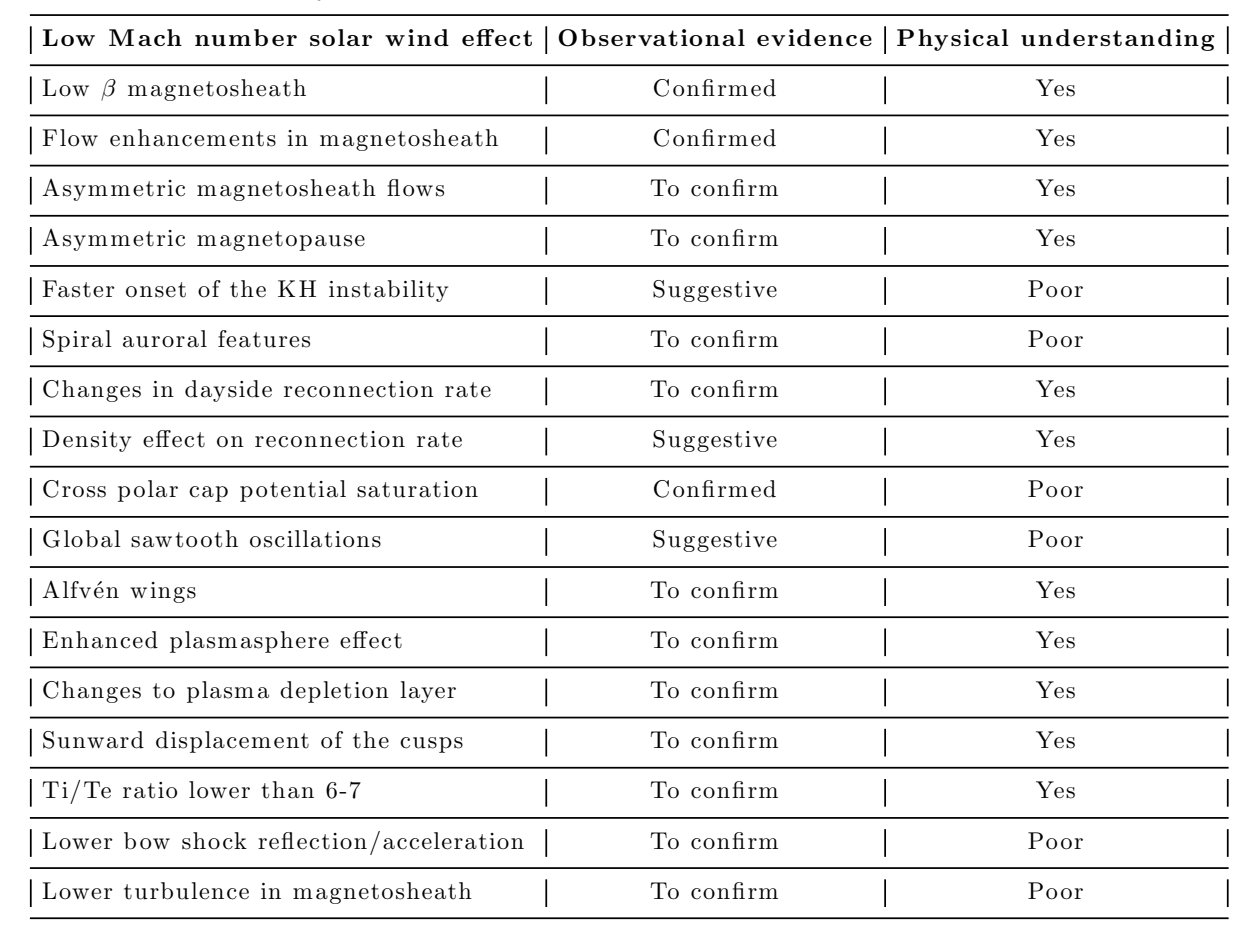

cumulative effect of strong driving; cf. O'Brien \& McPherron (2000) for a widely used ring current strength model.

\subsection{Impact of the solar wind Alfvén Mach number}

The prime role of the solar wind electric field must be weighted by other considerations. It was shown in particular that the solar wind Alfvén speed and Mach number can lead to very significant alterations of the solar wind-magnetosphere interaction (cf. Lavraud \& Borovsky 2008; Lopez et al. 2010; Siscoe 2011). Therefore, in addition to a large DawnDusk electric field, combined strong magnetic field and low densities (which lead to low Mach number values) have significant effects on how Earth's magnetosphere reacts to the driving, and such low Mach numbers typically occur during CMEs and MCs (Lavraud \& Borovsky 2008).

First, the solar wind Alfvén speed and Mach number are important because the Alfvén speed at meso-scales in the inflow region controls reconnection rate (e.g., Swisdak \& Drake 2007). The inclusion of this effect into a complex coupling function for the dayside magnetopause was performed by Borovsky (2008).

The Mach number also has important consequences on the global dynamics of Earth's magnetosheath, and in turn on the magnetopause shape and processes that occur there (Lavraud \& Borovsky 2008; Erkaev et al. 2012). The complex flow distributions and the changes to current systems that result from it then also impact global magnetospheric convection; i.e., low solar wind Mach numbers typically result in polar cap potential saturation (Lavraud \& Borovsky 2008; Lopez et al. 2010).

These and many other processes take place when low Mach number CMEs hit the Earth. A non-exhaustive list taken from Lavraud \& Borovsky (2008) is given in Table 


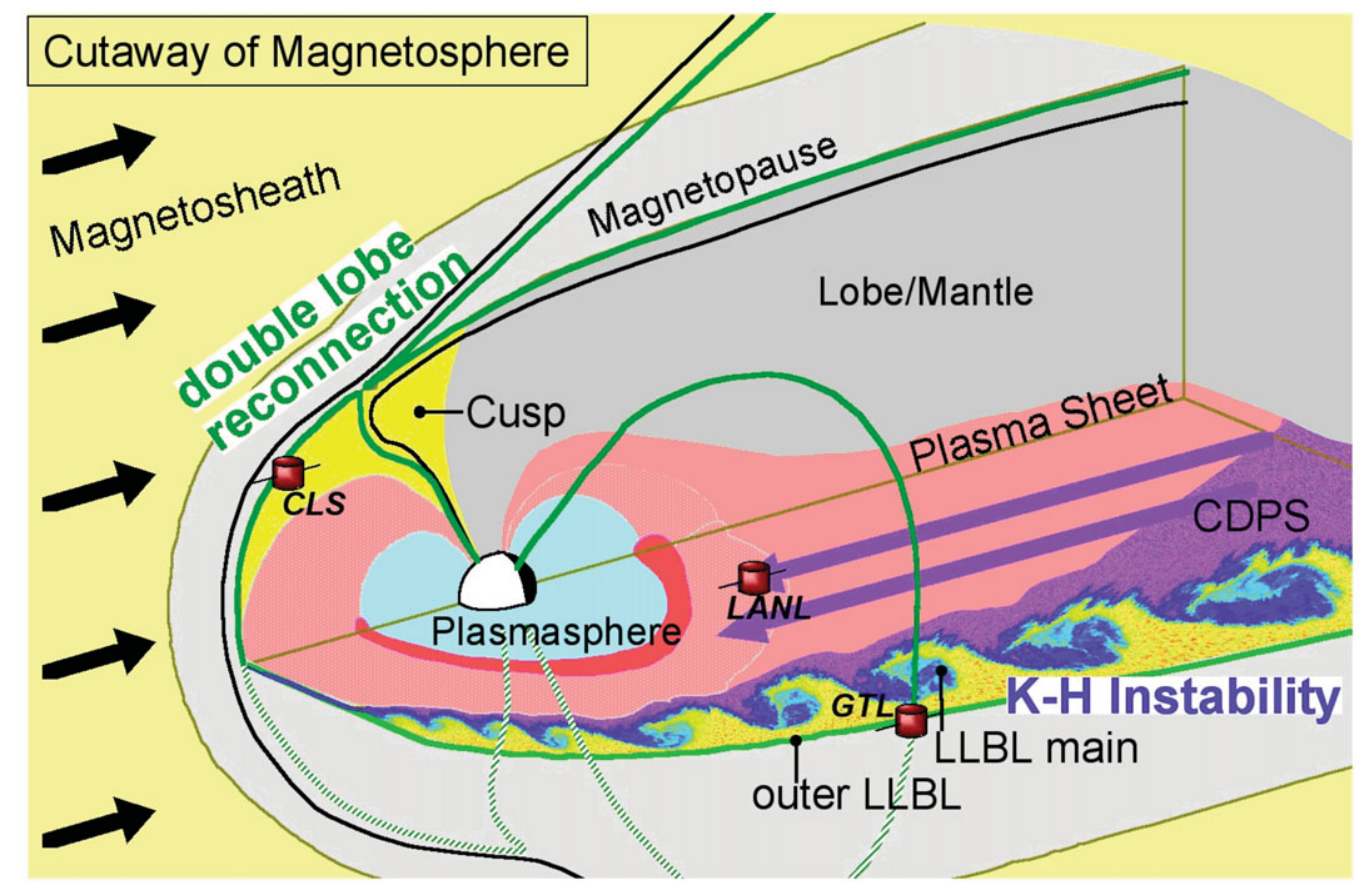

Figure 6. Structure of the magnetosphere with its main regions illustrated. Green field lines on the dayside depict the process known as double high-latitude reconnection, with a given northward-oriented IMF field line reconnecting in both the northern and southern hemisphere. This process creates newly closed flux tubes containing dense and cold plasma of solar wind origin, which may then be transported into the plasma sheet. An other CDPS formation mechanism is the Kelvin-Helmholtz (KH) instability. It is illustrated as waves on the flanks of the magnetopause. This mechanism may also allow direct entry of the cold and dense solar wind plasma into the magnetosphere. Figure courtesy of Kanako Seki (Nagoya University).

1. All these phenomena tend to occur simultaneously, so that solar wind-magnetosphere interaction is drastically altered as compared to the more common high Mach number interaction. Whether and how these effects come into play at other bodies is mostly unknown. It will be of particular interest to study Mach number effects at Mercury, with Messenger and Bepi-Colombo, since Mach numbers are typically lower closer to the Sun.

\subsection{Magnetospheric preconditioning mechanisms}

Specifics of the solar wind - magnetosphere interaction at Earth also include interesting processes known as pre-conditioning mechanisms. These arise from the fact that the state of the magnetosphere before strong driving changes how the system will react to that driving.

The first such process to mention is the formation of a cold and dense plasma sheet (CDPS) in the magnetotail. Such a CDPS may form during intervals of northwardoriented magnetic field. The processes responsible for the formation of the CDPS are depicted in Figure 6, and include primarily double high-latitude reconnection (Song et al. 2012) and the Kelvin-Helmhotz instability (e.g., Fujimoto \& Terasawa 1995). The importance of the formation of a CDPS then comes from the fact that a CDPS is capable of producing a stronger ring current during the first hours of an ensuing storm (cf. Lavraud \& Jordanova 2007 for details). The formation of a CDPS may easily occur just prior to CME arrival at Earth. 
An other preconditioning mechanism is mediated by the properties of the plasmasphere (cf. Figure 6), in terms of its spatial extent and density. Indeed, a fully formed plasmasphere will extend all the way to the magnetopause (in the form of plumes) during enhanced convection in storm time. Because the plasmasphere is made of extremely dense and cold plasma, if this plasma is entrained into the reconnection region at the dayside magnetopause it will substantially lower the Alfvén speed locally. As previously discussed, this will affect the local reconnection rate in such a way that reconnection is chocked. This effect was demonstrated through statistical analysis of magnetospheric response during plasmaspheric plume observation (Borovsky \& Denton 2006).

Other preconditioning mechanisms exist, for instance related to ring current and radiation belt, as well as to plasma transport in the magnetosphere or effects due to the composition of the plasma sheet as a function of ion outflows (e.g., with implications for reconnection rates in the magnetotail).

\section{Conclusions}

This review aimed to provide a discussion of our current knowledge of the processes that determine CME geo-effectiveness. There are many other additional properties and processes that have not been addressed due to space limitation. While key properties such as the CME trajectory, its magnetic field strength/orientation, its speed and their duration are first and largely set by the eruption processes at the Sun, highly influential processes also operate during propagation in the interplanetary medium and in the specific interaction with the object considered. Interplanetary processes in particular are capable of very significant alterations of CME geo-effectiveness (e.g., shock, compression, erosion). Those often are more than secondary in impact.

The impacts of each property and parameter still need to be fully understood and quantified. With regards to predictive capabilities, we note that among all the parameters that determine CME geo-effectiveness, predicting the North-South component of the magnetic field from solar data remains a key issue. As readers will notice, this review builds on the well-known work by Gosling (1993), the solar flare myth where he stated: "In this paper I outline a different paradigm of cause and effect that removes solar flares from their central position in the chain of events leading from the Sun to near-Earth space. Instead, this central role is given to events known as coronal mass ejections."

Acknowledgments. The authors wish to thank Brigitte Schmieder, Etienne Pariat, Jean-Marie Malherbe, and the other IAU300 conference organizers for the invitation to present at the conference and to write this review.

\section{References}

Akasofu, S.-I. 1981, Space Sci. Rev., 28, 2, 121

Borovsky, J. E. \& Denton, M. H. 2006, Geophys. Res. Lett., 33, L20101

Borovsky, J. E. 2008, J. Geophys. Res., 113, A8, A08228

Bothmer, V. \& Schwenn, R. 1998, Ann. Geophys., 16, 1

Burlaga, L. F. et al. 1982, Geophys. Res. Lett., 9, 1317

Burton, R. K., McPherron, R. L., \& Russell, C. T. 1975, J. Geophys. Res., 80, 4204

Cane, H. V., \& Richardson, I. G., St. Cyr, O. C. 2000, Geophys. Res. Lett., 27, 3591

Cargill, P. J., Chen, J., Spicer, D. S., \& Zalesak, S. T. 1995, Geophys. Res. Lett., 22, 647

Chen, J. 1996, J. Geophys. Res., 101, 27499

Dasso, S., Nakwacki, M. S., Demoulin, P., \& Mandrini, C. H. 2007, Sol. Phys., 244, 115

Delannée, C. \& Aulanier, G. 1999, Sol. Phys., 190, 107

Démoulin, P. \& Dasso, S. 2009, A\&A, 498, 551 
Dungey, J. W. 1961, Phys. Rev. Lett., 6, 47

Erkaev, N. V., et al. 2012, Geophys. Res. Lett., 39, L01103

Fenrich, F. R. \& Luhmann, J. G. 1998, Geophys. Res. Lett., 25, 2999

Fujimoto, M. \& Teresawa, T. 1995, J. Geophys. Res., 100, 12025

Gonzalez, W. D. \& Mozer, F. S. 1974, J. Geophys. Res., 79, 4186

Gopalswamy, N., Lara, A., Lepping, R. P., Kaiser, M. L., \& Berdichevsky, D., St. Cyr, O. C. 2000, Geophys. Res. Lett., 27, 145

Gopalswamy, N., Lara, A., Yashiro, S., Kaiser, M. L., \& Howard, R. A. 2001, J. Geophys. Res., 106, 29207

Gosling, J. T., Bame, S. J., McComas, D. J., \& Phillips, J. L. 1990, Geophys. Res. Lett., 17, 901

Gosling, J. T. 1993, J. Geophys. Res., 98, A11, 18937

Jian, L., Russell, C. T., Luhmann, J. G., \& Skoug, R. M. 2006, Sol. Phys., 239, 393

Lavraud, B. \& Jordanova, V. 2007, Geophys. Res. Lett., 34, L02102

Lavraud, B. \& Borovsky, J. E. 2008, J. Geophys. Res., 113, A00B08

Lavraud, B., Ruffenach, A., Kajdic, P., Manchester, W. B., \& Lugaz, N. 2013, J. Geophys. Res., submitted

Lopez, R. E., Bruntz, R., Mitchell, E. J., Wiltberger, M., Lyon, J. G., \& Merkin, V. G. 2010, J. Geophys. Res., 115, A12, A12216

Lugaz, N., Manchester, W. B., \& IV, Gombosi, T. I. 2005, ApJ, 634, 651

McComas, D., et al. 1988, ApJ, 93, 2519

Mulligan, T. \& Russell, C. T., Luhmann J. G. 1988, Geophys. Res. Lett., 25, 2959

Newell, P. T., Sotirelis, T., Liou, K., Meng, C.-I., \& Rich, F. J. 2007, J. Geophys. Res., 112, A01206

OBrien, T. P. \& McPherron, R. L. 2000, J. Geophys. Res., 105, 7707

Owens, M. J. 2006, J. Geophys. Res., 111, A12109

Owens, M. J., Merkin, V. G., \& Riley, P. 2006, J. Geophys. Res., 111, A03104

Perreault, P. \& Akasofu, S.-I. 1978, Geophys. J. R. Astr. Soc., 54, 547

Rouillard, A. P., et al. 2011, ApJ, 734, 7

Rouillard, A. P., et al. 2010, ApJ, 719, 1385

Rouillard, A. P., et al. 2009, Sol. Phys., 256, 307

Ruffenach, A., et al. 2012, J. Geophys. Res., 117, A9, A09101

Russell, C. T., et al. 2013, ApJ, 770, 38

Shepherd, S. G. 2007, J. Atmos. Solar-Terr. Phys., 69, 234

Siscoe, G. \& Crooker, N. 1974, Geophys. Res. Lett., 1, 17

Siscoe, G. 2011, J. Atmos. Solar-Terr. Phys., 73, 402

Subramanian, P., Lara, A., \& Borgazzi, A. 2012, Geophys. Res. Lett., 39, L19107

Swisdak, M. \& Drake, J. F. 2012, Geophys. Res. Lett., 34, L11106

Tenfjord, P. \& Østgaard, N. 2013, J. Geophys., Res., in press

Vrsnak, B. et al. 2010, A\&A, 512, A43

Wang, Y.-M. et al. 2000, J. Geophys. Res., 105, 25133

Zhang, J., Dere, K. P., Howard, R. A., \& Bothmer, V. 2003, ApJ, 582, 520

Zuccarello, F. P., Bemporad, A., Jacobs, C., Mierla, M., Poedts, S., \& Zuccarello, F. 2012, ApJ, 744,14 